УДК 517.5

DOI: $10.37069 / 1683-4720-2018-32-2$

\author{
(C)2018. Л.В. Вигівська
}

\title{
ЗАДАЧА ПРО ЕКСТРЕМАЛЬНЕ РОЗБИТТЯ КОМПЛЕКСНОЇ ПЛОЩИНИ З ФІКСОВАНИМИ ПОЛЮСАМИ НА КОЛІ
}

Дана робота присвячена дослідженню однієї екстремальної задачі з фіксованими полюсами. Це задача про добуток внутрішніх радіусів взаємно неперетинних симетричних областей відносно точок на одиничному колі на деяку додатну степінь внутрішнього радіуса області відносно початку координат.

MSC: $30 \mathrm{C} 75$.

Ключові слова: внутрішній радіус області, неперетинні області, фіксована система точок, розділяюче перетворення, квадратичний диференціал, функція Гріна.

\section{1. Вступ.}

Задачі про екстремальне розбиття почали свій розвиток з роботи М.О. Лаврентьєва [1], який поставив та розв'язав задачу про добуток конформних радіусів двох взаємно неперетинних областей. Екстремальними задачами для неперетинних областей з фіксованими полюсами в різні роки займались такі відомі математики як М.О. Лаврентьєв, Г. Грьотш, Г.М. Голузін, М.А. Лєбєдєв, П.П. Куфарев, А.Е. Фалес, Г.В. Кузьміна, В.М. Дубінін, Л.І. Колбіна, І.П. Мітюк, Ю.Є. Алєніцин, Дж.А. Дженкінс, М. Шиффер, П. Дюрен, З. Нехарі та ін.

\section{2. Позначення та означення.}

Нехай $\mathbb{N}$ - множина натуральних чисел, $\mathbb{C}$ - комплексна площина, $\overline{\mathbb{C}}=\mathbb{C} \bigcup\{\infty\}$ - сфера Рімана. Нехай $r(B, a)$ - внутрішній радіус області $B \subset \overline{\mathbb{C}}$, відносно точки $a \in B$. Внутрішній радіус області $B$ пов'язаний з узагальненою функцією Гріна $g_{B}(z, a)$ області $B$ наступними співвідношеннями

$$
\begin{gathered}
g_{B}(z, a)=-\ln |z-a|+\ln r(B, a)+o(1), \quad z \rightarrow a, \\
g_{B}(z, \infty)=\ln |z|+\ln r(B, \infty)+o(1), \quad z \rightarrow \infty .
\end{gathered}
$$

Набір точок $A_{n}=\left\{a_{k}\right\}_{k=1}^{n}, \quad k=\overline{1, n}$ будемо називати $n-$ променевою системою точок. В роботі будемо розглядати такі $a_{k}$, що $\left|a_{k}\right|=1, \quad k=\overline{1, n}$.

Нехай $P_{k}=P_{k}\left(A_{n}\right):=\left\{w: \arg a_{k}<\arg w<\arg a_{k+1}\right\}, a_{n+1}:=a_{1}, \alpha_{k}:=$ $\frac{1}{\pi} \arg \frac{a_{k+1}}{a_{k}}, \alpha_{n+1}:=\alpha_{1}, k=\overline{1, n}, \sum_{k=1}^{n} \alpha_{k}=2$.

Системою неперетинних областей називається скінченний набір довільних попарно неперетинних областей $\left\{B_{k}\right\}_{k=1}^{n}, n \in \mathbb{N}, n \geqslant 2$ таких, що $B_{k} \subset \overline{\mathbb{C}}, B_{k} \cap B_{m}=\emptyset$, $k \neq m, k, m=\overline{1, n}$.

Автор висловлює подяку професору О.К. Бахтіну за постановку задачі та цінні зауваження при написанні роботи. 


\section{3. Постановка задачі.}

Розглянемо наступну екстремальну задачу.

Нехай $\left\{B_{k}\right\}_{k=0}^{n}$ - довільна система взаємно неперетинних областей така, що $a_{0}=0 \in B_{0} \subset \overline{\mathbb{C}}, a_{k} \in B_{k} \subset \mathbb{C}, k=\overline{0, n},\left|a_{k}\right|=\left|e^{\frac{2 \pi k i}{n}}\right|=1, k=\overline{1, n}$, причому області $\left\{B_{k}\right\}_{k=1}^{n}-$ симетричні відносно одиничного кола.

Довести, що максимум функціонала

$$
J_{n}(\gamma)=r^{\gamma}\left(B_{0}, 0\right) \prod_{k=1}^{n} r\left(B_{k}, a_{k}\right)
$$

де $\gamma>1$, досягається для областей, які володіють $n$ - кратною симетрією.

\section{4. Основний результат.}

Теорема 1. Нехай $n \in \mathbb{N}, n \geqslant 2$. Тоді для довільного $\gamma \in\left(1 ; 0,5 n^{2}\right]$ ma довільного набора взаємно неперетинних областей $B_{k}$ таких, що $a_{0}=0 \in B_{0} \subset \overline{\mathbb{C}}$, $a_{k}=\exp \left(\frac{2 \pi k i}{n}\right) \in B_{k} \subset \mathbb{C}, k=\overline{0, n}$, причому області $B_{k}, k=\overline{1, n}-$ симетричні відносно одиничного кола, справедлива нерівність

$$
r^{\gamma}\left(B_{0}, 0\right) \prod_{k=1}^{n} r\left(B_{k}, a_{k}\right) \leqslant\left(\frac{4}{n}\right)^{n} \frac{\left(\frac{2 \gamma}{n^{2}}\right)^{\frac{\gamma}{n}}}{\left|1-\frac{2 \gamma}{n^{2}}\right|^{\frac{n}{2}+\frac{\gamma}{n}}}\left|\frac{n-\sqrt{2 \gamma}}{n+\sqrt{2 \gamma}}\right|^{\sqrt{2 \gamma}}
$$

знак рівності досягаеться коли $B_{k}^{(0)}, k=\overline{0, n}$ е круговими областями квадратичного диферениіала

$$
Q(w) d w^{2}=-\frac{\gamma w^{2 n}+2\left(n^{2}-\gamma\right) w^{n}+\gamma}{w^{2}\left(w^{n}-1\right)^{2}} d w^{2}
$$

Доведення. Зробимо розділяюче перетворення системи областей $B_{k}$ за допомогою функцій $\zeta=\left\{\pi_{k}(w)\right\}_{k=1}^{n}=\left(e^{-\frac{2 \pi k i}{n}} w\right)^{\frac{n}{2}}$, де вибрана така вітка цієї багатозначної функції, яка реалізує однолисте відображення кута $P_{k}$ на верхню півплощину.

Нехай $D_{k}^{(1)}, k=\overline{1, n}$, позначає область площини $\mathbb{C}_{z}$, отриману в результаті об'єднання зв'язної компоненти множини $\pi_{k}\left(B_{k} \bigcap \bar{P}_{k}\right)$, яка містить точку $\pi_{k}\left(a_{k}\right)=$ 1 , зі своїм симетричним відображенням відносно уявної осі. Позначимо через $D_{k}^{(2)}$, $k=\overline{1, n}$, таку область площини $\mathbb{C}_{z}$, яка отримана в результаті об'єднання зв'язної компоненти множини $\pi_{k}\left(B_{k+1} \bigcap \bar{P}_{k}\right)$, яка містить точку $\pi_{k}\left(a_{k+1}\right)=-1$, зі своїм симетричним відображенням відносно уявної осі, $B_{n+1}:=B_{1}, \pi_{n}\left(a_{n+1}\right):=\pi_{n}\left(a_{1}\right)$. Крім того, позначимо через $D_{k}^{(0)}$ таку область площини $\mathbb{C}_{z}$, отриману в результаті об'єднання зв'язної компоненти множини $\pi_{k}\left(B_{0} \bigcap \bar{P}_{k}\right)$, яка містить точку $z=0$, зі своїм симетричним відображенням відносно уявної осі. 3 визначення функцій $\pi_{k}$, випливає, що

$$
\left|\pi_{k}(w)\right| \sim\left|w_{k}\right|^{\frac{n}{2}}, \quad w \rightarrow 0, \quad w \in \overline{P_{k}},
$$




$$
\begin{gathered}
\left|\pi_{k}(w)-\pi_{k}\left(e^{\frac{2 \pi k i}{n}}\right)\right| \sim \frac{n}{2} \cdot\left|w-e^{\frac{2 \pi k i}{n}}\right|, \quad w \rightarrow e^{\frac{2 \pi k i}{n}}, \quad w \in \overline{P_{k}}, \\
\left|\pi_{k}(w)-\pi_{k}\left(e^{\frac{2 \pi(k+1) i}{n}}\right)\right| \sim \frac{n}{2} \cdot\left|w-e^{\frac{2 \pi(k+1) i}{n}}\right|, \quad w \rightarrow e^{\frac{2 \pi(k+1) i}{n}}, \quad w \in \overline{P_{k}} .
\end{gathered}
$$

Далі, використовуючи результати робіт [5,6] та симетрію структури траєкторій квадратичного диференціала, отримаємо нерівності

$$
\begin{gathered}
r\left(B_{0}, 0\right) \leqslant\left[\prod_{k=1}^{n} r^{\frac{4}{n^{2}}}\left(D_{k}^{(0)}, 0\right)\right]^{\frac{1}{2}}, \\
r\left(B_{k}, a_{k}\right) \leqslant\left[\frac{4}{n^{2}} r\left(D_{k}^{(1)}, 1\right) r\left(D_{k-1}^{(2)},-1\right)\right]^{\frac{1}{2}}, \quad k=\overline{1, n} .
\end{gathered}
$$

Отже, обчислюючи значення функціоналу $J_{n}(\gamma)$, отримаємо наступну оцінку

$$
\begin{gathered}
r^{\gamma}\left(B_{0}, 0\right) \prod_{k=1}^{n} r\left(B_{k}, a_{k}\right) \leqslant \\
\leqslant\left[\frac{4}{n^{2}}\right]^{\frac{n}{2}}\left[\prod_{k=1}^{n} r^{\gamma \frac{4}{n^{2}}}\left(D_{k}^{(0)}, 0\right) r\left(D_{k}^{(1)}, 1\right) r\left(D_{k}^{(2)},-1\right)\right]^{\frac{1}{2}}
\end{gathered}
$$

де $D_{k}^{(0)}, D_{k}^{(1)}, D_{k}^{(2)}$ - довільні неперетинні області такі, що $0 \in D_{k}^{(0)} \subset \mathbb{C}, 1 \in$ $D_{k}^{(1)} \subset \mathbb{C},-1 \in D_{k}^{(2)} \subset \mathbb{C}$.

Нехай

$$
\begin{gathered}
T_{k}:=\left\{z:(-1)^{k+1} \operatorname{Im} z>0\right\}, \quad k \in\{1,2\}, \\
G_{1}=\overline{T_{1}} \cap U_{1}, \quad G_{2}=\overline{\mathbb{C}} \backslash U_{1} \cap \overline{T_{1}}, \quad G_{3}=\overline{T_{2}} \cap U_{1}, \quad G_{4}=\overline{\mathbb{C}} \backslash U_{1} \cap \overline{T_{2}}, \\
\zeta=\beta(z)=\frac{2 z}{1+z^{2}} .
\end{gathered}
$$

3 визначення функцій $\beta(z)$ слідує, що

$$
\begin{gathered}
|\beta(z)| \sim 2|z|, \quad z \rightarrow 0, \quad z \in \overline{T_{k}}, \\
|\beta(z)-1| \sim \frac{1}{2}|z-1|^{2}, \quad z \rightarrow 1, \quad z \in \overline{T_{k}}, \\
|\beta(z)+1| \sim \frac{1}{2}|z+1|^{2}, \quad z \rightarrow-1, \quad z \in \overline{T_{k}} .
\end{gathered}
$$

Знову застосуємо розділяюче перетворення до областей $D_{0}, D_{1}, D_{2}$ відносно функції $\beta(z)$ та системи областей $\left\{\bar{G}_{k}\right\}_{k=1}^{4}$ позначимо через $\Omega_{0}^{(k)}, k=\overline{1,4}$; позначимо результат розділяючого перетворення областей $D_{j}, j \in\{1,2\}$, відносно функції $\beta(z)$ та системи областей $\left\{\bar{G}_{k}\right\}_{k=1}^{4}$ через області $\Omega_{1}^{(k)}, \Omega_{2}^{(k)}, k=\overline{1,4}$. 
Використовуючи результати робіт $[5,6]$ та симетрію областей $D_{0}, D_{1}, D_{2}$, справедливі співвідношення

$$
\begin{gathered}
r\left(D_{0}, 0\right) \leqslant\left[\frac{1}{2^{2}} r\left(\Omega_{0}^{(1)}, 0\right) \cdot r\left(\Omega_{0}^{(3)}, 0\right)\right]^{\frac{1}{2}} \\
r\left(D_{1}, 1\right) \leqslant\left[2^{4} r\left(\Omega_{1}^{(1)}, 1\right) r\left(\Omega_{1}^{(2)}, 1\right) r\left(\Omega_{1}^{(3)}, 1\right) r\left(\Omega_{1}^{(4)}, 1\right)\right]^{\frac{1}{8}}, \\
r\left(D_{2},-1\right) \leqslant\left[2^{4} r\left(\Omega_{2}^{(1)},-1\right) r\left(\Omega_{2}^{(2)},-1\right) r\left(\Omega_{2}^{(3)},-1\right) r\left(\Omega_{2}^{(4)},-1\right)\right]^{\frac{1}{8}} .
\end{gathered}
$$

Звідси маємо

$$
\begin{gathered}
r^{\gamma \frac{4}{n^{2}}}\left(D_{0}, 0\right) r\left(D_{1}, 1\right) r\left(D_{2},-1\right) \leqslant \\
\leqslant\left[\left(\left(\frac{1}{2}\right)^{2} r\left(\Omega_{0}^{(1)}, 0\right) \cdot r\left(\Omega_{0}^{(3)}, 0\right)\right)^{\frac{4 \gamma}{n^{2}}}\right]^{\frac{1}{2}} \times \\
\times\left[2^{4} r\left(\Omega_{1}^{(1)}, 1\right) r\left(\Omega_{1}^{(2)}, 1\right) r\left(\Omega_{1}^{(3)}, 1\right) r\left(\Omega_{1}^{(4)}, 1\right)\right]^{\frac{1}{8}} \times \\
\times\left[2^{4} r\left(\Omega_{2}^{(1)},-1\right) r\left(\Omega_{2}^{(2)},-1\right) r\left(\Omega_{2}^{(3)},-1\right) r\left(\Omega_{2}^{(4)},-1\right)\right]^{\frac{1}{8}} .
\end{gathered}
$$

Так як області $\Omega_{j}^{1}$, симетричні відносно одиничного кола областям $\Omega_{j}^{2}$, а області $\Omega_{j}^{3}$ симетричні $\Omega_{j}^{4}, j \in\{1,2\}$ то одержимо

$$
\begin{aligned}
r^{\gamma \frac{4}{n^{2}}}\left(D_{0}, 0\right) & r\left(D_{1}, 1\right) r\left(D_{2},-1\right) \leqslant\left[\frac{1}{2} r\left(\Omega_{0}^{(1)}, 0\right) \cdot \frac{1}{2} r\left(\Omega_{0}^{(3)}, 0\right)\right]^{\frac{2 \gamma}{n^{2}}} \times \\
& \times\left[\left(2 r\left(\Omega_{1}^{(1)}, 1\right)\right)^{2}\left(2 r\left(\Omega_{1}^{(3)}, 1\right)\right)^{2}\right]^{\frac{1}{8}} \\
\times & {\left[\left(2 r\left(\Omega_{2}^{(1)},-1\right)\right)^{2}\left(2 r\left(\Omega_{2}^{(3)},-1\right)\right)^{2}\right]^{\frac{1}{8}} . }
\end{aligned}
$$

Остаточно одержимо таку нерівність

$$
\begin{gathered}
r^{\gamma \frac{4}{n^{2}}}\left(D_{0}, 0\right) r\left(D_{1}, 1\right) r\left(D_{2},-1\right) \leqslant \\
\leqslant 2^{1-\frac{4 \gamma}{n^{2}}}\left\{\left[r^{\frac{8 \gamma}{n^{2}}}\left(\Omega_{0}^{(1)}, 0\right) r\left(\Omega_{1}^{(1)}, 1\right) r\left(\Omega_{2}^{(1)},-1\right)\right]^{\frac{1}{4}}\right\} \times \\
\times\left\{\left[r^{\frac{8 \gamma}{n^{2}}}\left(\Omega_{0}^{(3)}, 0\right) r\left(\Omega_{1}^{(3)}, 1\right) r\left(\Omega_{2}^{(3)},-1\right)\right]^{\frac{1}{4}}\right\} .
\end{gathered}
$$


Переходячи до максимуму правої частини нерівності (4) отримаємо, що

$$
\begin{gathered}
r^{\frac{8 \gamma}{n^{2}}}\left(\Omega_{0}^{(3)}, 0\right) r\left(\Omega_{1}^{(3)}, 1\right) r\left(\Omega_{2}^{(3)},-1\right) \leqslant \\
r^{\frac{8 \gamma}{n^{2}}}\left(E_{0}, 0\right) r\left(E_{1}, 1\right) r\left(E_{2},-1\right),
\end{gathered}
$$

де $E_{0}, E_{1}, E_{2}$ - кругові області квадратичного диференціала

$$
-\frac{\left(n^{2}-2 \gamma\right) z^{2}+2 \gamma}{z^{2}\left(z^{2}-1\right)^{2}} d z^{2}
$$

причому $0 \in E_{0}, 1 \in E_{1},-1 \in E_{2}$.

Оскільки величина $\frac{8 \gamma}{n^{2}} \leqslant 4$ при всіх $\gamma \in\left(1, \frac{n^{2}}{2}\right]$, то використовуючи результат роботи [6], справедлива рівність

$$
\begin{gathered}
r^{\frac{8 \gamma}{n^{2}}}\left(E_{0}, 0\right) r\left(E_{1},-1\right) r\left(E_{2}, 1\right)= \\
=2^{\frac{8 \gamma}{n^{2}}+6} \cdot\left(\frac{2 \sqrt{2 \gamma}}{n}\right)^{\frac{8 \gamma}{n^{2}}} \cdot\left(2-\frac{2 \sqrt{2 \gamma}}{n}\right)^{-\frac{1}{2}\left(2-\frac{2 \sqrt{2 \gamma}}{n}\right)^{2}} \cdot\left(2+\frac{2 \sqrt{2 \gamma}}{n}\right)^{-\frac{1}{2}\left(2+\frac{2 \sqrt{2 \gamma}}{n}\right)^{2}}= \\
=2^{2} \cdot\left(\frac{2 \sqrt{2 \gamma}}{n}\right)^{\frac{8 \gamma}{n^{2}}} \cdot\left(1-\frac{2 \gamma}{n^{2}}\right)^{-2-\frac{4 \gamma}{n^{2}}}\left[\frac{1-\frac{\sqrt{2 \gamma}}{n}}{1+\frac{\sqrt{2 \gamma}}{n}}\right]^{\frac{4}{n} \sqrt{2 \gamma}} .
\end{gathered}
$$

Підставимо одержану величину в (4), маємо

$$
\begin{gathered}
r^{\gamma \frac{4}{n^{2}}}\left(\Omega_{0}, 0\right) r\left(\Omega_{1}, 1\right) r\left(\Omega_{2},-1\right) \leqslant \\
\leqslant 2^{2-\frac{4 \gamma}{n^{2}}}\left(\frac{2 \sqrt{2 \gamma}}{n}\right)^{\frac{4 \gamma}{n^{2}}} \cdot\left(1-\frac{2 \gamma}{n^{2}}\right)^{-1-\frac{2 \gamma}{n^{2}}}\left[\frac{1-\frac{\sqrt{2 \gamma}}{n}}{1+\frac{\sqrt{2 \gamma}}{n}}\right]^{\frac{2}{n} \sqrt{2 \gamma}} .
\end{gathered}
$$

Тепер залишилось підставити праву частину останньої рівності в (3), маємо

$$
\begin{aligned}
& r^{\gamma}\left(B_{0}, 0\right) \prod_{k=1}^{n} r\left(B_{k}, a_{k}\right) \leqslant \\
& \leqslant\left[\frac{4}{n^{2}}\right]^{\frac{n}{2}}\left[2^{2-\frac{4 \gamma}{n^{2}}}\left(\frac{2 \sqrt{2 \gamma}}{n}\right)^{\frac{4 \gamma}{n^{2}}} \cdot\left(1-\frac{2 \gamma}{n^{2}}\right)^{-1-\frac{2 \gamma}{n^{2}}}\left[\frac{1-\frac{\sqrt{2 \gamma}}{n}}{1+\frac{\sqrt{2 \gamma}}{n}}\right]^{\frac{2}{n} \sqrt{2 \gamma}}\right]^{\frac{n}{2}}= \\
& =\left(\frac{4}{n}\right)^{n} \cdot \frac{\left(\frac{2 \gamma}{n^{2}}\right)^{\frac{\gamma}{n}}}{\left(1-\frac{2 \gamma}{n^{2}}\right)^{\frac{n}{2}+\frac{\gamma}{n}}} \cdot\left(\frac{1-\frac{\sqrt{2 \gamma}}{n}}{1+\frac{\sqrt{2 \gamma}}{n}}\right)^{\sqrt{2 \gamma}}
\end{aligned}
$$

де $B_{k}^{(0)}, a_{k}, k=\overline{0, n}, a_{0}=0,-$ кругові області та полюси квадратичного диференціала (2), відповідно. 


\section{5. Наслідки з теореми 1.}

Наслідок 1. Нехай $n \in \mathbb{N}, n \geqslant 2$. Тоді для довільного $\gamma \in\left(1 ; 0,5 n^{2}\right]$ та довільного набора взаємно неперетинних областей $B_{k}$ таких, що $a_{0}=0 \in B_{0} \subset \overline{\mathbb{C}}$, $a_{k}=\exp \left(\frac{2 \pi k i}{n}\right) \in B_{k} \subset \mathbb{C}, k=\overline{0, n}$, причому області $B_{k}, k=\overline{1, n}$ - симетричні відносно одиничного кола, справедлива нерівність

$$
r^{\gamma}\left(B_{0}, 0\right) \prod_{k=1}^{n} r\left(B_{k}, a_{k}\right) \leqslant r^{\gamma}\left(B_{0}^{(0)}, 0\right) \prod_{k=1}^{n} r\left(B_{k}^{(0)}, a_{k}\right),
$$

де $B_{k}^{(0)}, k=\overline{0, n}$ є круговими областями квадратичного диференціала

$$
Q(w) d w^{2}=-\frac{\gamma w^{2 n}+2\left(n^{2}-\gamma\right) w^{n}+\gamma}{w^{2}\left(w^{n}-1\right)^{2}} d w^{2} .
$$

Наслідок 2. Нехай $n \in \mathbb{N}, n \geqslant 2$. Тоді для довільного $\gamma \in\left(1 ; 0,5 n^{2}\right]$ та довільного набора взаємно неперетинних областей $B_{k}$ таких, що $a_{0}=0 \in B_{0} \subset \overline{\mathbb{C}}$, $a_{k}=R \cdot \exp \left(\frac{2 \pi k i}{n}\right) \in B_{k} \subset \mathbb{C}, R>0, k=\overline{0, n}$, причому області $B_{k}, k=\overline{1, n}-$ симетричні відносно кола радіуса $R$, справедлива нерівність

$$
r^{\gamma}\left(B_{0}, 0\right) \prod_{k=1}^{n} r\left(B_{k}, a_{k}\right) \leqslant\left(\frac{4}{n}\right)^{n} \frac{\left(\frac{2 \gamma}{n^{2}}\right)^{\frac{\gamma}{n}}}{\left|1-\frac{2 \gamma}{n^{2}}\right|^{\frac{n}{2}+\frac{\gamma}{n}}}\left|\frac{n-\sqrt{2 \gamma}}{n+\sqrt{2 \gamma}}\right|^{\sqrt{2 \gamma}} \cdot R^{\gamma+n},
$$

знак рівності досягається коли $B_{k}^{(0)}, k=\overline{0, n}$ є круговими областями квадратичного диференціала

$$
Q(w) d w^{2}=-\frac{\gamma w^{2 n}+2\left(n^{2}-\gamma\right) R^{n} w^{n}+R^{2 n} \gamma}{w^{2}\left(w^{n}-R^{n}\right)^{2}} d w^{2} .
$$

Наслідок 3. Нехай $n \in \mathbb{N}, n \geqslant 2$. Тоді для довільного $\gamma \in\left(1 ; 0,5 n^{2}\right]$ та довільного набора взаємно неперетинних областей $B_{k}$ таких, що $a_{0}=0 \in B_{0} \subset U$, $a_{k}=\exp \left(\frac{2 \pi k i}{n}\right) \in B_{k} \subset \mathbb{C}, k=\overline{0, n}$, причому області $B_{k}, k=\overline{1, n}$ - симетричні відносно одиничного кола, справедлива нерівність

$$
r^{\gamma}\left(B_{0}, 0\right) \prod_{k=1}^{n} r\left(B_{k}, a_{k}\right) \leqslant\left(\frac{4}{n}\right)^{n} \frac{\left(\frac{2 \gamma}{n^{2}}\right)^{\frac{\gamma}{n}}}{\left|1-\frac{2 \gamma}{n^{2}}\right|^{\frac{n}{2}+\frac{\gamma}{n}}}\left|\frac{n-\sqrt{2 \gamma}}{n+\sqrt{2 \gamma}}\right|^{\sqrt{2 \gamma}}
$$

знак рівності досягається коли $B_{k}^{(0)}, k=\overline{0, n}$ є круговими областями квадратичного диференціала

$$
Q(w) d w^{2}=-\frac{\gamma w^{2 n}+2\left(n^{2}-\gamma\right) w^{n}+\gamma}{w^{2}\left(w^{n}-1\right)^{2}} d w^{2}
$$




\section{Цитована література}

1. Лаврентъев М.А. К теории конформных отображений// Тр. Физ.-мат. ин-та АН СССР. 1934. - 5. - C. 159-245.

2. Тамразов П.М. Экстремальные конформные отображения и полюсы квадратичных дифференциалов // Известия АН СССР, серия мат. - 1968. - 32, № 5. - С. 1033-1043.

3. Бахтин А., Бахтина Г., Зелинский Ю. Тополого-алгебраические структуры и методы в комплексном анализе// Праці ін-ту мат-ки НАН України. - 2008. - 308 с.

4. Выговская Л.В. О проблемме В.Н. Дубинина для симметричных многосвязных областей// Український математичний вісник. - 2017. - Т. 14, № 2. - С. 295-302.

5. Дубинин В.Н. Разделяющее преобразование областей и задачи об экстремальном разбиении// Зап. науч. сем. Ленингр. отд-ния Мат. ин-та АН СССР. - 1988. - 168. - С. 48-66.

6. Дубинин В.Н. Метод симметризации в геометрической теории функций комплексного переменного// Успехи мат. наук. - 1994. - 49 (295), № 1. - С. 3-76.

\section{References}

1. Lavrent'ev, M.A (1934). On the theory of conformal mappings. Travaux Inst. Physico-Math. Stekloff Acad. Sci. USSR, 5, 159-245.

2. Tamrazov, P.M. (1968). Extreme conformal mappings and poles quadratic differentials. Izv. Akad. Nauk SSSR Ser. Mat., 32(5), 1033-1043 (in Russian). Translation in (1968) Math. USSR-Izv., 2(5), 987-996.

3. Bakhtin, A.K., Bakhtina, G.P., Zelinskii, Yu.B. (2008). Topological-algebraic structures and geometric methods in complex analysis. Proceedings of the Institute of Mathematics of NAS of Ukraine (in Russian).

4. Vyhivska, L. (2018). On the problem of V.N. Dubinin for symmetric multiply connected domains. J. Math. Sci., 229(1), 108-113.

5. Dubinin, V.N. (1988). The separating transformation of domains and problems on the extremal partition. Analytical theory of numbers and theory of functions. Part 9, Zap. Nauchn. Sem. LOMI, 168, 48-46 (in Russian). Translation in (1991) J. Soviet Math., 53(3), 252-263.

6. Dubinin, V.N. (1994). Symmetrization in the geometric theory of functions of a complex variable. Uspekhi Mat. Nauk, 49(1), 3-76 (in Russian). Translation in (1994) Russian Math. Survey, 49(1), 1-79.

\section{L.V. Vyhivska}

The problem of extreme decomposition of a complex plane with fixed poles on a circle.

The problem of extreme decomposition of a complex plane with fixed poles on a circle. Investigation on geometric function theory has been conducted by several researchers, however, few studies have reported on the problem considering extremal configurations the product of inner radii of non-overlapping domains with respect to fixed poles. The paper describes the problem of finding the maximum of the product of inner radii of mutually non-overlapping symmetric domains with respect to points on a unit circle multiply by a certain positive degree $\gamma$ of the inner radius of the domain with respect to the zero. The problem was studied using the method of separating transformation. Proving the theorem shows that the maximum is obtained if $\gamma \in\left(1, n^{2}\right]$ and for all $n \geqslant 2$. Its results and the method for the obtaining of these results can be used in the theory of potential, approximations, holomorphic dynamics, estimation of the distortion problems in conformal mapping, and complex analysis.

Keywords: inner radius of domain, non-overlapping domains, fixed system of points, separating transformation, quadratic differential, Green's function. 
Задача про екстремальне розбиття комплексної площини з фіксованими полюсами на колі

\section{Л.В. Выговская}

Задача об экстремальном разбиении комплексной плоскости с фиксированными полюсами на окружности.

Данная работа посвящена исследованию одной экстремальной задачи с фиксированными полюсами. Это задача о произведении внутренних радиусов взаимно неналегающих симметричных областей относительно точек на единичной окружности на некоторую положительную степень внутреннего радиуса области относительно начала координат.

Ключевые слова: внутренний радиус области, неналегающие области, фиксированная система точек, разделяющее преобразование, квадратичный дифференциал, функция Грина.

Інститут математики НАН України, Київ

Отримано 19.05.18

lyudmilavygivska@ukr. net 